\title{
MODEL-THEORETIC CHARACTERIZATIONS OF ARCS AND SIMPLE CLOSED CURVES
}

\author{
PAUL BANKSTON
}

(Communicated by Thomas J. Jech)

\begin{abstract}
Two compact Hausdorff spaces are co-elementarily equivalent if they have homeomorphic ultracopowers; equivalently if their Banach spaces of continuous real-valued functions have isometrically isomorphic Banach ultrapowers (or, approximately satisfy the same positive-bounded sentences). We prove here that any locally connected compact metrizable space co-elementarily equivalent with an arc (resp. a simple closed curve) is itself an arc (resp. a simple closed curve). The hypotheses of metrizability and local connectedness cannot be dropped.
\end{abstract}

0. Introduction. An arc is a topological space homeomorphic with the closed unit interval I on the real line; a simple closed curve (s.c.c.) is a space homeomorphic with the standard unit circle $S$ in the plane. By a continuum we mean a connected compact Hausdorff space; a locally connected metrizable continuum is termed a Peano continuum. (Peano continua are precisely those Hausdorff spaces that are continuous images of $I$, by the Hahn-Mazurkiewicz theorem [12].) A simple triod is any space homeomorphic with the set $\{\langle x, y\rangle:-1 \leq x \leq 1$ and $y=$ 0 , or $x=0$ and $0 \leq y \leq 1\}$ in the plane. A point of a connected space is a cut point if the complement of that point is disconnected. Our topological nomenclature is predominately from Willard $[\mathbf{1 2}]$.

Characterizations of $I$ and $S$ go back to the 1920 's, principally to the work of R. L. Moore.

0.1 THEOREM (MOORE [12]). If $X$ is a metrizable continuum with exactly two noncut points, then $X$ is an arc.

0.2 THEOREM (MOORE [12]). If $X$ is a nondegenerate metrizable continuum such that the complement of any two points is disconnected, then $X$ is a s.c.c.

0.3 TheOREM (MOORE [11]). If $X$ is a nondegenerate Peano continuum containing no simple triod, then $X$ is either an arc or a s.c.c.

The first model-theoretic characterization of $I$ and $S$ of which we are aware appears in the paper [10] by C. W. Henson, C. J. Jockusch, L. A. Rubel, and G. Takeuti. For any space $X$, let $F(X)$ be its bounded lattice of closed sets. We use standard model-theoretic terminology, as can be found in [6].

Received by the editors June 13, 1987 and, in revised form, April 6, 1988. Presented at the Annual Meeting of the Association for Symbolic Logic, New York City, December 1987.

1980 Mathematics Subject Classification (1985 Revision). Primary 03C20, 03C65, 54B25, 54D05, 54D35, 54F25, 54F65.

Key words and phrases. co-elementary equivalence, compact Hausdorff spaces, Peano continua, ultracoproducts, arcs, simple closed curves. 
0.4 THEOREM (HENSON ET AL. [10]). If $X$ is a metrizable space such that $F(X)$ is elementarily equivalent with $F(I)$ (resp. with $F(S)$ ), then $X$ is an arc (resp. a s.c.c.).

The form of Theorem 0.4 is interesting in itself; it characterizes a member of a class of spaces, within that class, using a list ("taxonomy") of model-theoretically specified topological properties. (We treat this topic more fully in [5].) What makes this theorem more than just a routine application of Theorems 0.1 and 0.2 is that compactness is not assumed for $X$.

Our result is similar to Theorem 0.4 in form but not in content. We characterize the same spaces within a smaller class (the locally connected compact metrizable spaces) using a different taxonomy. Our methods, which employ Theorem 0.3 (Theorems 0.1 and 0.2 seem to be useless for our purposes), dc not allow us to drop compactness; however, there are examples to show that metrizability and local connectedness are essential.

The taxonomy arises as follows. For a compact Hausdorff space $X$, let $C(X)$ be the Banach space of continuous real-valued functions (with the norm of uniform convergence). The first-order alphabet appropriate to $C(X)$, introduced by $\mathrm{C}$. W. Henson [9], includes vector addition, a unary operation of scalar multiplication for each rational scalar, the null vector, and two unary predicates $P$ and $Q$. The interpretation of $P x$ (resp. $Q x$ ) is that the norm of $x$ is $\leq 1$ (resp. $\geq 1$ ). The positive-bounded formulas are built up from the atomic formulas using disjunction, conjunction, and quantification bounded over members of the unit ball. For each positive-bounded formula $\phi$ and whole number $n \geq 1$, the formula $\phi_{n}$ is the $n$th approximation to $\phi$ and is defined by induction on the complexity of $\phi$. If $\phi$ is atomic, say it is $x=y$, then $\phi_{n}$ is $P m(x-y)$. If $\phi$ is $P x$ (resp. $Q x$ ) then $\phi_{n}$ is $P\left(1-\frac{1}{n}\right) x$ (resp. $\left.Q\left(1+\frac{1}{n}\right) x\right)$. Finally, $(\phi \wedge \psi)_{n}$ is $\phi_{n} \wedge \psi_{n},(\phi \vee \psi)_{n}$ is $\phi_{n} \vee$ $\psi_{n},(\exists x(P x \wedge \phi))_{n}$ is $\exists x\left(P x \wedge \phi_{n}\right)$, and $(\forall x(P x \rightarrow \phi))_{n}$ is $\forall x\left(P x \rightarrow \phi_{n}\right)$. Given a structure $A$ and a positive-bounded sentence $\sigma$, we say $\mathcal{A}$ approximately satisfies $\sigma$ (in symbols $A \vDash_{A} \sigma$ ) if $A$ satisfies $\sigma_{n}$ for every $n \geq 1$. Our taxonomy is then the positive-bounded sentences; the topological property represented by such a sentence $\sigma$ is just $\left\{X: X\right.$ is compact Hausdorff and $\left.C(X) \vDash_{A} \sigma\right\}$.

The main result of $[9]$ is that two Banach spaces $A$ and $B$ approximately satisfy the same positive-bounded sentences if and only if $A$ and $B$ have isometrically isomorphic Banach ultrapowers. (Briefly, one forms the Banach ultraproduct $\Pi_{D}^{B} A_{i}$ by first taking the usual ultraproduct, removing the elements of infinite norm, and then identifying two elements if they are infinitely close.) In the case where the Banach spaces are of the form $C(X)$ for $X$ compact Hausdorff, the Banach-Stone theorem [7] allows one to recover the topological structure of $X$ from the Banach space structure of $C(X)$. In [9], Henson shows that the Banach ultrapower $\Pi_{D}^{B} C(X)$ is of the form $C(\hat{X})$, where $\hat{X}$ is a suitable compact Hausdorff space. In [2, 3, 4] we study the ultracoproduct construction $\Sigma_{D} X_{i}$ for compact Hausdorff spaces $X_{i}$; it turns out that the ultracopower $\Sigma_{D} X$ is precisely Henson's $\hat{X}$. In general, we have $C\left(\Sigma_{D} X_{i}\right)$ isometrically isomorphic with $\Pi_{D}^{B} C\left(X_{i}\right)$. Putting these facts together, we obtain the result that for any two compact Hausdorff spaces $X$ and $Y$, $C(X)$ and $C(Y)$ approximately satisfy the same positive-bounded sentences (i.e. $X$ and $Y$ belong to the same taxon in this particular taxonomy), if and only if $C(X)$ and $C(Y)$ have isometrically isomorphic Banach ultrapowers, if and only if 
$X$ and $Y$ have homeomorphic ultracopowers. When this last condition holds, we say $X$ and $Y$ are co-elementarily equivalent and write $X \equiv Y$.

There are several equivalent ways of defining the ultracoproduct $\Sigma_{D} X_{i}$, each with its particular merits. To give but three, we have:

(i) the maximal ideal space of $\Pi_{D}^{B} C\left(X_{i}\right)$ (with the natural ring structure);

(ii) $p^{-1}[D]$, where $p: \beta\left(\bigcup_{i} X_{i}\right) \rightarrow \beta(I)$ is the Stone-Čech lifting of the map that takes an element of the disjoint union to its index in $I$; and

(iii) the compactification of the topological ultraproduct (see [1]) using ultraproducts of zero sets from the factors as a Wallman basis (see $[\mathbf{3}, \mathbf{8}]$ for details, as well as other ways to view $\Sigma_{D} X_{i}$ ).

The approach (iii) to the ultracoproduct allows the most flexibility. Because each $X_{i}$ is compact Hausdorff, one may substitute any closed set basis $B_{i}$ of $X_{i}$ in place of the zero sets, as long as $B_{i}$ is a sublattice of $F\left(X_{i}\right)$. (One can easily see that $B_{i}$ will then be a Wallman basis satisfying the condition that disjoint members of $F\left(X_{i}\right)$ can be separated by disjoint members of $B_{i}$. This is more than enough to ensure that the compactification using the Wallman basis $\Pi_{D} B_{i}$ will result in $\Sigma_{D} X_{i}$.)

The relation $\equiv$ is an equivalence relation; the co-elementary equivalence of $X$ and $Y$ follows from the elementary equivalence of Wallman bases $B_{X}$ and $B_{Y}$ for $X$ and $Y$ respectively (taken as lattices: use the Keisler-Shelah ultrapower theorem). If $X$ and $Y$ are 0-dimensional compact Hausdorff spaces, then $X \equiv Y$ if and only if their Boolean algebras of closed open sets are elementarily equivalent (see [3]).

In [3] we conjectured that if $X$ is any compact metrizable space co-elementarily equivalent with $I$, then $X$ is an arc. In [8], however, R. Gurevic disproves that conjecture with the following.

0.5 EXAMPLE (GUREVIČ [8]). There is a metrizable continuum $X$ such that $X \equiv I$, but $X$ fails to be locally connected.

Co-elementary equivalence detects connectedness; Gurevic shows that it fails to detect local connectedness. In fact, he proves that if $D$ is any nonprincipal ultrafilter on the natural numbers $\omega$, then $\Sigma_{D} I$ cannot be locally connected. He uses this fact, plus a downward Löwenheim-Skolem type argument, to produce his Example 0.5.

The new result in this article is the following.

0.6 THEOREM. Let $X$ be any locally connected compact metrizable space coelementarily equivalent with I (resp. S). Then $X$ is an arc (resp. a s.c.c.).

The proof, an outgrowth of techniques developed in [3 and 4], is continua-theoretic, making crucial use of Moore's Theorem 0.3. Before proceeding to the proof, we include an example, kindly suggested by the referee to show that metrizability is an indispensable hypothesis in Theorem 0.6.

0.7 EXAMPLE (REFEREE). There is a locally connected continuum $X$ such that $X \equiv I$, but $X$ fails to be metrizable.

CONSTRUCTION. Let $T$ be the first order theory of dense linear orderings with distinct endpoints. If $A$ is a model of $T$, let $\mathcal{L}_{A}$ be the lattice of all finite unions of closed intervals of $A$. $\mathcal{L}_{A}$ forms a closed set basis for the associated linear order space, which we denote $X_{A}$. If $A$ is a complete ordering, then $X_{A}$ is connected, 
locally connected, compact Hausdorff, and $\mathcal{L}_{A}$ is a Wallman basis that can be used to form the ultracopowers of $X_{A}$.

Now let $T^{\prime}$ be the set of all first order sentences of lattice theory that are true in all lattices $\mathcal{L}_{A}$, where $A$ is a model of $T$. Given a model $\mathcal{L}$ of $T^{\prime}$, let $A$ be the set of atoms of $\mathcal{L}$. It is easy to show that there are two linear orderings on $A$, one the reverse of the other and both models of $T$, such that $\mathcal{L} \cong \mathcal{L}_{A}$ where $A$ is either one of these orderings. Since $T$ is $\aleph_{0}$-categorical, so is $T^{\prime}$. Consequently, if $A$ and $B$ are any two models of $T$, then $\mathcal{L}_{A}$ and $\mathcal{L}_{B}$ are elementarily equivalent. So let $A$ and $B$ be complete models of $T$, say $A$ is the ordering on the closed unit interval and $B$ is the ordering on the long line with last element. Then $X_{A}=I$ is metrizable, while $X_{B}$ is not. However, $\mathcal{L}_{A}$ and $\mathcal{L}_{B}$ are elementarily equivalent; hence they have isomorphic ultrapowers. Therefore $X_{A}$ and $X_{B}$ are co-elementarily equivalent.

1. Proof of Theorem 0.6. As remarked above, if $\left\langle X_{i}: i \in I\right\rangle$ is a collection of compact Hausdorff spaces and $D$ is an ultrafilter on $I$, then the ultraproduct of closed set lattices $\Pi_{D} F\left(X_{i}\right)$ represents a Wallman basis for the closed subsets of $\Sigma_{D} X_{i}$. In particular, if $A_{i}$ and $B_{i}$ are closed subsets of $X_{i}$ for each $i \in I$, then the sequences $\left\langle A_{i}: i \in I\right\rangle$ and $\left\langle B_{i}: i \in I\right\rangle$ represent the same closed subset of $\Sigma_{D} X_{i}$ if and only if $\left\{i: A_{i}=B_{i}\right\} \in D$. We denote this basic closed set as $\Sigma_{D} A_{i}$; the singletons $\Sigma_{D} x_{i}=\Sigma_{D}\left\{x_{i}\right\}$ correspond exactly to the points of the topological ultraproduct, which densely embeds in $\Sigma_{D} X_{i}$. It is easy to see that $\Sigma_{D}(\cdot)$ commutes with finite unions and intersections.

Let $P$ be any topological property. We say $P$ is "preserved by co-elementary equivalence" if whenever $X$ and $Y$ are compact Hausdorff, $X \equiv Y$, and $X$ has property $P$, then $Y$ also has property $P$. Easy to prove (see [3]) is the fact that the properties of finiteness and connectedness are preserved by co-elementary equivalence. (Local connectedness and metrizability are not so preserved, as a consequence of Examples 0.5 and 0.7. Also, since there is a sentence $\phi$ in the first order language of bounded lattices such that if $X$ is any compact Hausdorff space, then $X$ is locally connected if and only if $F(X) \vDash \phi$, we see by Example 0.5 that the co-elementary equivalence of metrizable continua $X$ and $Y$ does not necessarily imply that $F(X)$ and $F(Y)$ are elementarily equivalent.)

We will need the following fact (which also played an important role in [4]).

1.1 LEMMA (GUREVIČ $[4,8])$. Let $\left\langle X_{i}: i \in I\right\rangle$ be compact Hausdorff spaces. For $i \in I$, let $x_{i} \in X_{i}$, with $C_{i}$ the (connected) component of $x_{i}$ in $X_{i}$. Then $\Sigma_{D} C_{i}$ is the component of $\Sigma_{D} x_{i}$ in $\Sigma_{D} X_{i}$.

A key property that proved useful in [4] in the counting of co-elementary equivalence classes, and that will prove useful here, is " $n$-odicity". Let $X$ be compact Hausdorff, $n<\omega$. An $n$-wheel on $X$ is a cover $\{K\} \cup\left\{L_{j}: j<n\right\}$ of $X$ by subcontinua such that:

(i) $K \backslash \bigcup_{j<n} L_{j} \neq \varnothing(K$ is the "hub");

(ii) $L_{j} \backslash K \neq \varnothing$ for $j<n\left(L_{j}\right.$ is a "spoke");

(iii) $L_{j} \cap K \neq \varnothing$ for $j<n$; and

(iv) for $j<k<n, L_{j} \cap L_{k}=\varnothing$.

Of course, if $X$ has an $n$-wheel then $X$ is a continuum. $X$ is $n$-odic if $X$ has an $n$-wheel but no $m$-wheel for $m>n$. Note that simple triods are 3-odic, arcs 
are 2-odic, simple closed curves are 1-odic, and that the 0-odic continua are the indecomposable continua. Note also that higher dimensional cells, such as $I^{2}$, have $n$-wheels for all $n<\omega$. The following uses Lemma 1.1.

1.2 LEMMA [4]. The property of being $n$-odic for any $n<\omega$ is preserved by co-elementary equivalence.

We say that a compact Hausdorff $X$ contains an $n$-wheel if $X$ has a subcontinuum that has an $n$-wheel. Obviously arcs and simple closed curves contain no 3-wheels.

The main new result we require to prove Theorem 0.6 is the following.

1.3 LEMMA. Suppose $X$ and $Y$ are co-elementarily equivalent compact Hausdorff spaces, and that $X$ is locally connected and contains an $n$-wheel. Then $Y$ also contains an $n$-wheel.

Proof. Assume $D$ and $\mathcal{E}$ are ultrafilters, and that $h: \Sigma_{D} X \rightarrow \Sigma_{\mathcal{E}} Y$ is a homeomorphism. Let $\{K\} \cup\left\{L_{j}: j<n\right\}$ be an $n$-wheel on $X$. An $n$-wheel is called fat if, in clauses (i)-(iii) of the definition above, the intersections are not only nonempty, but have nonempty interiors.

Now while the original $n$-wheel may not be fat, we may "fatten" the hub and spokes using a compactness argument so that the resulting family $\left\{K^{\prime}\right\} \cup\left\{L_{j}^{\prime}: j<\right.$ $n$ \} would be a fat $n$-wheel if the new sets were only connected. In this we are in luck: since $X$ has a basis of connected open sets, we may easily arrange for the sets $K^{\prime}, L_{j}^{\prime}, j<n$, to be connected as well. So without loss of generality, we may assume that our original $n$-wheel $\{K\} \cup\left\{L_{j}: j<n\right\}$ is fat, and we see immediately that $\left\{\Sigma_{D} K\right\} \cup\left\{\Sigma_{D} L_{j}: j<n\right\}$ is a fat $n$-wheel in $\Sigma_{D} X$. Let $K^{\prime}=h\left[\Sigma_{D} K\right], L_{j}^{\prime}=$ $h\left[\Sigma_{D} L_{j}\right], j<n$. Then, since $\mathrm{h}$ is a homeomorphism, $\left\{K^{\prime}\right\} \cup\left\{L_{j}^{\prime}: j<n\right\}$ is a fat $n$-wheel in $\Sigma_{\varepsilon} Y$.

Since $K^{\prime} \backslash \bigcup_{j<n} L_{j}^{\prime}$ has nonempty interior, there is a point

$$
\Sigma_{D} x_{i} \in K^{\prime} \backslash \bigcup_{j<n} L_{j}^{\prime}
$$

Similarly, for $j<n$, there is a point $\Sigma_{\varepsilon} y_{j, i} \in L_{j}^{\prime} \backslash K^{\prime}$. Using a second compactness argument, one can show that if $C$ and $D$ are any disjoint closed subsets of $\Sigma_{\mathcal{E}} Y$, there are, for $i \in I$, open sets $U_{i}, V_{i} \subseteq Y$, with disjoint closures, such that $C \subseteq \Sigma_{\mathcal{E}} \bar{U}_{i}$ and $D \subseteq \Sigma_{\varepsilon} \bar{V}_{i}$. Of course, $\Sigma_{\mathcal{E}} \bar{U}_{i} \cap \Sigma_{\mathcal{E}} \bar{V}_{i}=\varnothing$. Hence, for each $j<n$, we can find closed $\Sigma_{\varepsilon} C_{j, i} \supseteq L_{j}^{\prime}$ such that $\Sigma_{\varepsilon} C_{j, i} \cap \Sigma_{\varepsilon} C_{k, i}=\varnothing, j<k<n$, and $\Sigma_{\varepsilon} x_{i} \notin$ $\bigcup_{j<n} \Sigma_{\varepsilon} C_{j, i}$. Using Lemma 1.1, let $\Sigma_{\varepsilon} L_{j, i}$ be the component of $\Sigma_{\varepsilon} C_{j, i}$ containing $\Sigma_{\varepsilon} y_{j, i}, j<n$. Then $L_{j}^{\prime}$, being connected, must be contained in $\Sigma_{\varepsilon} L_{j, i}$. Similarly we obtain an ultracoproduct subcontinuum $\Sigma_{\varepsilon} K_{i} \supseteq K$ containing no point $\Sigma_{\varepsilon} y_{j, i}$. Thus the family $\left\{\Sigma_{\mathcal{E}} K_{i}\right\} \cup\left\{\Sigma_{\mathcal{E}} L_{j, i}: j<n\right\}$ is an $n$-wheel in $\Sigma_{\mathcal{E}} Y$ consisting of ultracoproduct subcontinua. From this we easily infer that $\left\{i:\left\{K_{i}\right\} \cup\left\{L_{j, i}: j<n\right\}\right.$ is an $n$-wheel in $Y\} \in D$; whence $Y$ contains an $n$-wheel.

Now we are ready to finish the proof of Theorem 0.6. Assume $X$ is locally connected compact metrizable, and suppose $X \equiv I$. Since $I$ is connected and infinite, so is $X$. Since $I$ is 2-odic, so too is $X$, by Lemma 1.2. $S$ is 1-odic, so $X$ cannot be a s.c.c. $X$ is a nondegenerate Peano continuum which is not a s.c.c. Hence, by Theorem 0.3 , all we need to show is that $X$ contains no simple triod. 
Suppose the contrary. Since simple triods are 3-odic, $X$ contains a 3-wheel. By Lemma 1.3, so does $I$. This is a clear contradiction, and we are done. The argument in which $S$ replaces $I$, and vice versa, is similar.

1.4 REMARKS AND QUESTIONS. (i) Theorem 0.6 is a characterization theorem in which a space $X$ is characterized within a class $K$ of spaces via $\equiv$. (Equivalently, via the $C$-taxonomy described earlier. This topic is more fully explored in [5].) The definition of $\Sigma_{D} X_{i}$ makes sense for arbitrary Tichonov spaces, and in [3] it is proved that $\Sigma_{D} X_{i}$ and $\Sigma_{D} \beta\left(X_{i}\right)$ are naturally homeomorphic. Thus every finite space is characterized within the class of Tichonov spaces via $\equiv$ (where the relevant taxonomy is now the $C^{*}$-taxonomy in which only bounded continuous functions are considered). Clearly no infinite space can be so characterized, but there are many interesting variations on the question. For example, since every Tichonov space is co-elementarily equivalent with a compact metrizable space $[\mathbf{4}, \mathbf{8}]$, it follows that no noncompact Tichonov $X$ can be characterized via $\equiv$ in a class $K$ of Tichonov spaces which contains $X$ as well as all compact metrizable spaces. Is every Tichonov space co-elementarily equivalent with a noncompact metrizable space? A positive answer to this would yield a host of negative results about characterizability. In particular, it would be interesting to see whether compactness is an essential hypothesis in Theorem 0.6.

(ii) We would like to broaden our inventory of locally connected compact metrizable spaces $M$ that can be substituted for $I$ and $S$ in Theorem 0.6. This can easily be done to a small extent; $M$ can be any finite disjoint union of points, arcs and simple closed curves. Beyond this trivial generalization, however, it seems that new techniques are called for. At present we do not even know whether $M$ can be a simple triod, let alone a finite 1-complex. As for higher dimensional Peano continua, there are well-known analogues of Theorems 0.1 and 0.2 characterizing 2-cells and 2-spheres, enabling Theorem 0.4 to be broadened to include 2-complexes [5]. However, these analogues seem to suffer from the same lack of applicability in our situation because they involve cut (and noncut) points. (See, e.g., Remark 3.2.11 in [3].)

(iii) While it is relatively easy to construct pairs of nonhomeomorphic metrizable continua that are co-elementarily equivalent, the problem becomes more difficult (and as yet unsolved) when we further require local connectedness. Especially in light of Theorem 0.6, it is a natural (and indeed inevitable) question whether there are any Peano continua that cannot be characterized via $\equiv$ within the class of locally connected compact metrizable spaces.

ACKNOWLEDGEMENT. The author would like to thank the referee not only for Example 0.7, which enhanced the mathematical content of this paper, but for helpful comments which substantially improved its readability.

\section{REFERENCES}

1. P. Bankston, Ultraproducts in topology, General Topology Appl. 7 (1977), 283-308.

2. __ Expressive power in first order topology, J. Symbolic Logic 49 (1984), 478-487.

3. __ Reduced coproducts of compact Hausdorff spaces, J. Symbolic Logic 52 (1987), 404-424.

4. __ Co-elementary equivalence for compact Hausdorff spaces and compact abelian groups, J. Pure Appl. Algebra (to appear).

5. _ Taxonomies of model-theoretically defined topological properties (submitted).

6. C. C. Chang and H. J. Keisler, Model theory, North-Holland, Amsterdam, 1973. 
7. N. Dunford and J. T. Schwartz, Linear operators, Part I, Interscience, New York, 1966.

8. R. Gurevix, On ultracoproducts of compact Hausdorff spaces, J. Symbolic Logic 53 (1988), 294-300.

9. C. W. Henson, Nonstandard hulls of Banach spaces, Israel J. Math. 25 (197), 108-144.

10. C. W. Henson, C. G. Jockusch, Jr., L. A. Rubel, and G. Takeuti, First order topology, Dissertationes Math. 143 (1977), 1-40.

11. R. L. Moore, Foundations of point set theory, Amer. Math. Soc. Colloq. Publ., vol. 13, Providence, R.I., 1962.

12. S. Willard, General topology, Addison-Wesley, New York, 1970.

Department of MAthematics, Statistics and Computer SCience, MarquetTe UNIVERSITY, MILWAUKEE, WISCONSIN 53233 